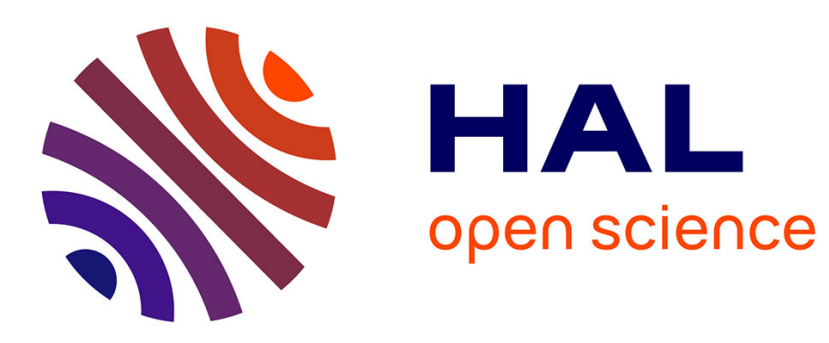

\title{
Three-dimensional luminescence microscopy for quantitative plasma characterization in bulk semiconductors
}

\author{
A Wang, A Das, J Hermann, D Grojo
}

\section{- To cite this version:}

A Wang, A Das, J Hermann, D Grojo. Three-dimensional luminescence microscopy for quantitative plasma characterization in bulk semiconductors. Applied Physics Letters, 2021, 119 (4), pp.041108. 10.1063/5.0059431 . hal-03350812

\section{HAL Id: hal-03350812 \\ https://hal.science/hal-03350812}

Submitted on 21 Sep 2021

HAL is a multi-disciplinary open access archive for the deposit and dissemination of scientific research documents, whether they are published or not. The documents may come from teaching and research institutions in France or abroad, or from public or private research centers.
L'archive ouverte pluridisciplinaire HAL, est destinée au dépôt et à la diffusion de documents scientifiques de niveau recherche, publiés ou non, émanant des établissements d'enseignement et de recherche français ou étrangers, des laboratoires publics ou privés. 


\section{Three-dimensional luminescence microscopy for quantitative plasma characterization in bulk semiconductors}

A. Wang, ${ }^{1, \text { a) }}$ A. Das, ${ }^{1}$ J. Hermann, ${ }^{1}$ and D. Grojo ${ }^{1, \text { b) }}$

Aix-Marseille Université, CNRS, LP3, UMR7341, 13288 Marseille, France

(Dated: 16 July 2021)

Important challenges remain in the development of ultrafast laser writing inside semiconductor materials because the properties of narrow gap materials cause strong propagation distortions to intense infrared light. Here, we introduce a simple and robust imaging method for high-dynamic-range investigations of the laser-matter interactions in bulk semiconductors. Supported by measurements in gallium arsenide and silicon, we show how $z$-scan imaging of the band-to-band radiative recombination enables quantitative reconstruction of the three-dimensional distributions of freecarriers generated by nonlinear ionization with ultrashort pulses. The validity is confirmed by comparisons with ultrafast transmission microscopy (shadowgraphy) images. The superior sensitivity of the zero-background luminescence method allows the measurement of local carrier densities as low as $\approx 10^{16} \mathrm{~cm}^{-3}$ inside GaAs that is inaccessible by shadowgraphy. It provides the first direct evidence of the low density plasma generated far prior to the focus that causes the previously reported intensity clamping phenomenon. The potential of this non-coherent 3D imaging method to assess complex beam distortion features is also exemplified by real-time pre-compensation of aberration for an intense interacting beam.

Intense infrared lasers can be focused to induce micro/nano-scale permanent modifications anywhere inside semiconductor materials. ${ }^{1,2}$ This holds potential to integrate new functions on single microelectronic chips including microfluidic cooling circuits $^{2}$, waveguides ${ }^{3-6}$ or other optical components ${ }^{7}$. For these applications, a key is to obtain highly localized laser energy deposition. However, the strong nonlinearities inherent to narrow bandgap materials lead to beam distortions and more easily ionized materials in the pre-focal region that strongly deteriorates the achievable point spread function and the achievable 3D writing performances ${ }^{1,8-11}$. A direct way to monitor the spatial features of nonlinear ionization inside semiconductors is then highly desirable for improved understanding of these limiting aspects and subsequent optimization studies. ${ }^{1,12,13}$

There are numerous surface techniques, including electrical measurements, to evaluate free-carrier concentrations in semiconductor materials ${ }^{14}$. However, most of them are inappropriate for the transient local excitations deep inside materials corresponding 3D laser writing configurations. Directly inspired by similar studies in dielectrics, ultrafast pump-probe transmission (shadowgraphy) $)^{8,9}$ and interferometric microscopy techniques ${ }^{1,8}$ have been attempted in the infrared for retrieval of the changes of the complex optical permittivity due to local carrier injection with focused pulses. These measurements give directly $2 \mathrm{D}$ projection images of the generated plasmas but 3D tomographic reconstruction is only conceivable under simplifying hypotheses with the use of appropriate numerical procedures (e.g. Abel inversion ${ }^{15}$ ). In addition, the transmission or phase change induced by the micro-plasma is hardly resolvable when the produced carrier densities are orders of magnitude below the critical plasma density. In practice, this leads to a lower limit for the dynamic range of in-

${ }^{a)}$ Electronic mail: andong.wang@univ-amu.fr

b) Author to whom correspondence should be addressed. Electronic mail david.grojo@univ-amu.fr vestigation of about $10^{18} \mathrm{~cm}^{-3}$ at best. ${ }^{9,16,17}$ Limited by these drawbacks, there have been so far no direct observations of low density plasmas in the prefocal regime despite their importance on the achievable peak energy density as extrapolated by measurements ${ }^{1,11,13,18}$ and simulations for $\mathrm{Si}^{10,11,19}$ On the other hand, laser-induced photoemission shows strong potential for studying characteristics of intense laser local excitation and defect formation in bulk dielectrics ${ }^{20-23}$. However, there has been no equivalent demonstration by luminescence diagnostics inside semiconductors to date.

In this paper, we demonstrate $3 \mathrm{D}$ reconstruction of freecarrier distributions inside semiconductors generated with focused ultrashort pulses by taking direct advantage of the associated photoemission phenomena. We identify and filter radiative band-to-band recombination in $\mathrm{GaAs}$ but also $\mathrm{Si}$ despite the expected low yield in indirect band gap materials. Supported by comparisons with ultrafast shadowgraphy images, we show how this luminescence signal can be calibrated for reliable plasma density measurements. Case studies on GaAs demonstrate the superior dynamic range of this method that enables the first direct measurement of low-density prefocal plasma. We show also how the non-coherent nature of the signal suppresses artefacts limiting the 3D imaging capabilities of pump-and-probe technologies.

Our experimental arrangement is shown in the Fig. 1(a). Femtosecond laser pulses of 190-fs pulse duration (Pharos, Lightconversion) are delivered by an optical parametric amplifier (Orpheus, Lightconversion) operated at 1550-nm wavelength and tightly focused $300 \mu \mathrm{m}$ under the surface of samples using an objective lens (Olympus $100 \times$, numerical aperture (NA) 0.85, marked 'Lens1' in the figure) corrected for spherical aberrations. As illustrated with Fig. 1(a), we add an infrared microscopy imaging system to observe laterally laser interactions inside GaAs. It is based on an arrangement of a long working distance objective (Mitutoyo $20 \times$, NA0.40, marked 'Lens2' in the figure), a tube lens, and a camera (Raptor, OWL640). The imaging focus defined by the distance $d$ with respect to sample surface (lateral $x$-axis) is adjustable by 


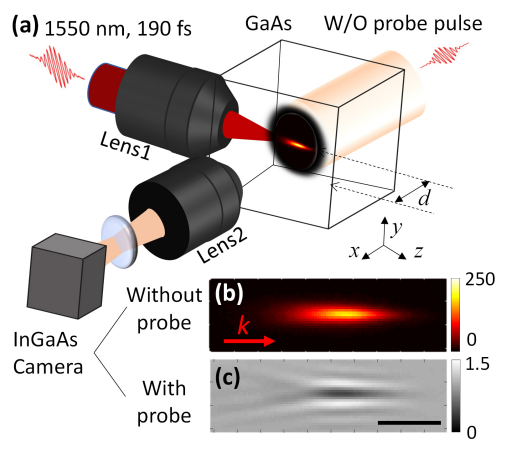

FIG. 1. (a) Schematic of the experimental geometry. (b) Photoemission image of the interaction region inside GaAs as measured without probe illumination. $\mathrm{k}$ vector represents the laser propagation direction. (c) Pump-and-probe transmission image for the same interaction conditions measured at sub-picosecond delay. The pump pulse energy is $16.8 \mathrm{~nJ}$. Scale bar: $20 \mu \mathrm{m}$

moving the sample holder. A typical emission image crosssectioning the focal region of the pump is shown in Fig. 1(b). The exposure time of the camera is $40 \mathrm{~ms}$ so that the emission is integrated over 400 interacting pulses according to the $10 \mathrm{kHz}$ repetition rate of the laser source. The pseudo-color scale directly represents the pixel signal value that is proportional to the emission amplitude.

The emission image is compared to the corresponding ultrafast shadowgraphy image presented in Fig. 1(c). This measurement uses a probe pulse from the same laser system and delayed by $\approx 500$ fs to capture the maximum electron density just after the excitation. ${ }^{9,17}$ The grey-scale image is normalized with a reference image to directly represent transmission of the excited region ( $T=1$ at rest). It is noteworthy to mention at this stage a difference between the two images. The shadowgraphy displays not only losses (transmission smaller than 1) due to inverse Bremsstrahlung absorption by free carriers in the laser-generated plasma, ${ }^{9,17}$ but also local transmissions that are exceeding 1. As we will see later on, this artefact, absent in the luminescence image (Fig. 1(b)), is attributed to diffraction features caused by the inherent coherence of the laser illumination.

An excellent spatial correlation is observed between the luminescence and shadowgraphy images displayed in Fig. 1 (b) and (c), respectively. This directly indicates that the light emission originates from free carriers generated by the focused laser pulse. To assess the physical origin of the luminescence, we first investigated the polarization properties of the emission that was found to be non-polarized, excluding thus a significant contribution from pump laser beam scattering. This is also confirmed by spectral measurements made by focusing the emission onto a fiber connected to a spectrometer (ULS4096CL-EVO, Avantes). The measured emission spectrum peaking near $900 \mathrm{~nm}$ is shown in Fig. 2(a). Interestingly, we show with an analysis made in Fig. 2(b) that the
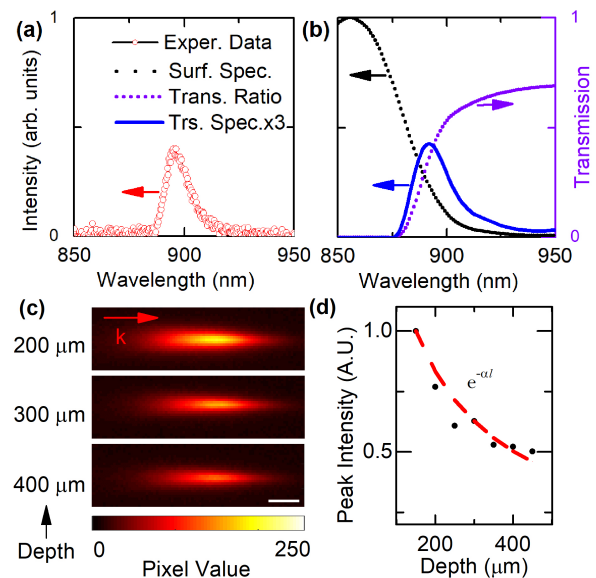

FIG. 2. (a) Measured emission spectrum from laser excitation $300 \mu \mathrm{m}$ inside GaAs; (b) Simulated spectrum (blue solid line) obtained by the product of GaAs surface luminescence (black dotted line, from Ref. ${ }^{24}$ ) and GaAs transmission spectrum to account for signal filtering before free-propagation in Air (purple dashed line, from Ref. ${ }^{25}$ ). (c) Photoemission images measured for excitation at three different depths. Scale bar: $20 \mu \mathrm{m}$. The k vector represents the pump propagation direction. (d) Peak emission intensity measured as a function of depth compared to expected Beer-Lambert's Law.

line shape compares very favorably with literature data on radiative band-to-band recombination from surface studies, provided that we account for the absorption edge filtering caused by the thickness of GaAs crossed by the signal in this in-depth measurement (blue curve in Fig. 2(b)). While bremsstrahlung or blackbody emission could have been considered, the excellent agreement obtained on the position and dissymmetry of the line shape with these considerations is taken as evidence of a signal dominated by radiative recombination.

We have chosen to concentrate here on GaAs, a direct narrow gap semiconductor material $(E=1.44 \mathrm{eV}, \lambda=860 \mathrm{~nm})$, to rely on a relatively strong radiative band-to-band recombination signal in the near-visible domain. However, we have checked that the same method can be applied to other semiconductors, provided that appropriate sensing instruments (spectral response and sensitivity) are used. This holds for $\mathrm{Si}$, which is a low emission yield indirect gap material $(E=$ $1.1 \mathrm{eV}, \lambda=1.1 \mu \mathrm{m}$ ), as exemplified with Fig. S1 showing a similar imaging and spectral analysis made with InGaAsbased infrared sensor technologies.

According to the Beer-Lambert's law, the transmitted emission intensity, can be increased by simply reducing the measuring depth $d$. Fig. 2(c) shows emission images of the same interaction produced at three different position changing $d$. We note that the signal intensity increases as $d$ decreases. As shown with Fig. 2(d) the maximum intensity of the images follows well the Beer-Lambert's law considering the absorption coefficient $\alpha=34 \mathrm{~cm}^{-1}$ for GaAs taken at the wave- 

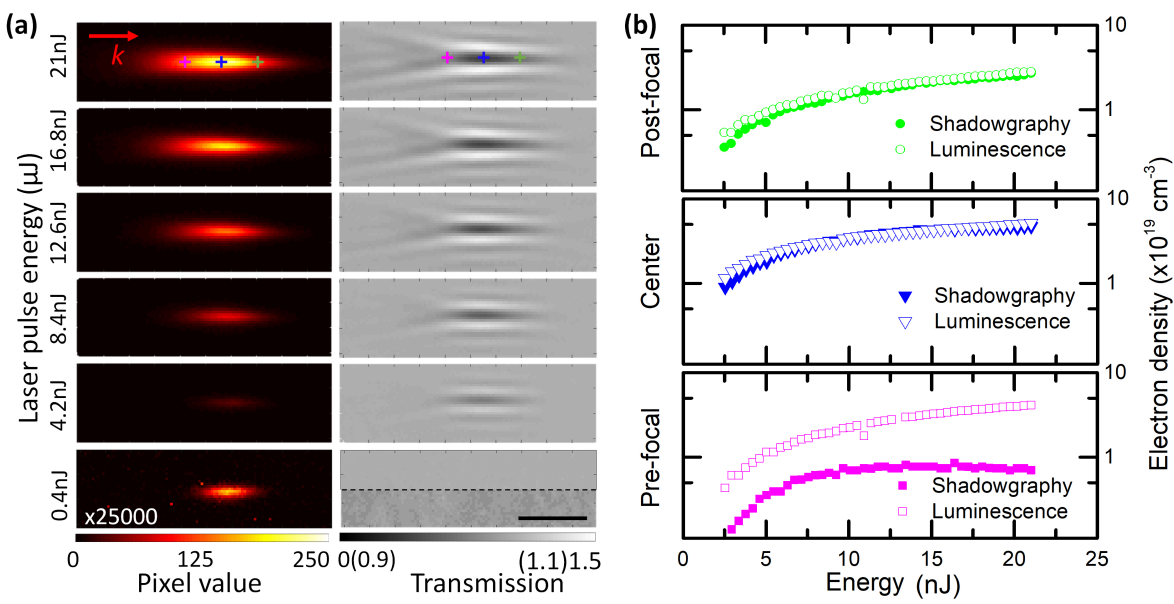

FIG. 3. (a) Luminescence (left column) and shadowgraphy (right column) images captured for different incident pulse energies. For the pulse energy of $0.4 \mathrm{~nJ}$, the luminescence signal is magnified for 25000 times by increasing the exposure time to $10 \mathrm{~s}$ and the digital gain of the camera to $20 \mathrm{~dB}$. The scale bar of the lower-half of the shadowgraphy image is rescaled between 0.9 and 1.1, showing no noticeable signal at the noise detection limit. The scale bar is $10 \mu \mathrm{m}$. (b) Comparison of measured electron densities obtained by the shadowgraphy (solid symbols) and the luminescence methods (Hollow symbol) as a function of applied pulse energy at three positions of the plasma (marked by crosses with corresponding colors in (a)).

length of $895 \mathrm{~nm}$. The overall agreements in Fig. 2 confirm the phenomenon of the emission can be properly described by the band-to-band photoluminescence. For technological considerations, it highlights the importance of reducing the imaging depth when signal-to-noise ratio becomes an issue. This aspect can be essential for low emission yield materials as $\mathrm{Si}$ that is mentioned before.

After identifying the origin of the emission, we show now how the captured images can be used for quantitative analysis of the free-carrier concentrations. According to the recombination statistics in non-doped semiconductors, ${ }^{26}$ the expected photon emission rate and the measured signal from direct band-to-band transition is proportional to the square of the produced free-carrier density $n_{e}$. his simple relationship holds for the relatively modest carrier densities measured in this report (validity confirmed up to typical $10^{19} \mathrm{~cm}^{-3}$ ) but will reasonably deviate from measured emissions when high energy density effects come into play and affect the carrier relaxation response (e.g. Auger recombination, temperature change). In this paper, we use this simple relationship for the measured signal on each pixel,

$$
S_{\text {pix }}=C_{\text {calib }} \times n_{e}^{2}
$$

where $C_{\text {calib }}$ is a constant that can be derived from a reference measurement. Alternatively, it can be calculated from the material physical properties, imaging geometry and sensor specifications according to the product

$$
C_{\text {calib }}=\tau \times R \times T \times \Omega \times V \times N \times C_{\text {cam }}
$$

where $\tau$ and $R$ are respectively the free-carrier lifetime and the radiative recombination probability of the material, $T$ represents the transmission ratio of the imaging process (considering all the losses from the plasma to camera, like the absorption and reflection of the GaAs layer, and the loss of the imaging system, etc.), $\Omega$ and $V$ accounts for solid angle collection and integrated volume of emission defined by the $N A$ and magnification of the observing microscope $M, N$ represents the pulse numbers, and $C_{c a m}$ describes the conversion rate from incoming photons to pixel-counts. The detailed calculations of each parameter are given in Supplementary Note 1.

For a calibration from a reference measurement, we can use the comparison between the shadowgraphy and the luminescence images shown in Fig. 1. Looking at the minimum transmission (corresponding to maximum luminescence) and applying the Drude model (see details in Supplementary Note 2), we associate to this region to a carrier density of $2.5 \times 10^{19} \mathrm{~cm}^{-3}$ from the shadowgraphy image. The corresponding luminescence signal leads to $C_{\text {calib }}=2.3 \times 10^{-37}$ pixel counts $\mathrm{cm}^{-6}$ as a calibration constant used in the rest of this work. For a calibration according to Eq. 2, by taking $N A=0.4, M=20$, a pixel size of $15 \mu \mathrm{m}, C_{\text {cam }}=1.1 \times$ $10^{-3}$ counts/incoming photon on camera pixels and $\tau=1 \mathrm{~ns}$ and $R=1.7 \times 10^{-10} \mathrm{~cm}^{3} \cdot \mathrm{s}^{-1}$ that are typical numbers reported for $\mathrm{GaAs}^{14,27}$, we find a very similar calibration constant, $C_{\text {calib }}=2.9 \times 10^{-37}$ pixel counts $\cdot \mathrm{cm}^{-6}$. This shows the validity of our calibration. Important for the applicability of the method, this indicates also that quantitative measurements are always possible without the need for a reference measure- 
ment, provided the characteristics of the measuring system and the material properties are known.

To evaluate the robustness and reliability of the calibration depending on the studied conditions, we show with Fig. 3 a space-resolved analysis for different incident pulse energies. Fig. 3(a) compares luminescence (left panel) and shadowgraphy (right panel) images for gradually decreasing pulse energies. To compare the carrier density derived from these two methods, we select three representative positions of the plasma that are in pre-focal region, centered (maximum signal), and post-focal, respectively. The positions are marked by cross symbols of different colors in Fig. 3(a). The results obtained according to Eq. 1 for the luminescence images and the Drude model applied to the shadowgrahs are shown in Fig. 3(b). Interestingly, the measured electron density strongly deviates from the power law expected at low exciation levels ${ }^{28}$ and shows a clear saturation with increasing pulse energy. This results from propagation nonlinearities and corresponds well with more indirect observations made in recent works ${ }^{1}$.

Comparing the results obtained by these two methods, a remarkable agreement is found for the regions of best focus and post-focal independently off the laser pulse energy. However, an important deviation is observed in the pre-focal region where the electron density evaluated by shadowgraphy is much smaller than the value deduced from luminescence. The difference can be attributed to different effects integrated by the two methods. The shadowgraphy method relies on the measurement of a probe that has not seen only interacted with the imaged plane, but is transmitted through the whole plasma (lateral $x$-dimension). Obviously, the integration of large and non-uniform plasmas may affect the validity of our simple Drude model analysis leading to an estimate of the averaged density in the apparent excited regions. ${ }^{9,17}$ Also, as discussed before with Fig.1(c), the probe light not only endures the absorption accounted in our model but also diffraction effects due to the local refractive index changes induced by the plasma. These effects, clearly revealed by changing image focus as shown in Fig. S2, create a bias on the analysed absorption signal that cannot be suppressed unless complex in-line holography numerical procedures are used to account for al propagation aspects. On the contrary, the non-coherent nature of luminescence suppress interference or diffraction effects. While other effects as self-absorption or some other plasma refractive effects would need to be rigorously accounted, the measured emission is longitudinally integrated only over the depth of field of the imaging system. This leads to a more robust and accurate method for local measurements in these non-uniform plasma conditions.

Other benefits of the zero-background luminescence method are in the sensitivity and dynamic-range. Theoretically, the sensitivity limit can be improved without limitation by increasing the number of emission events integrated in time (exposure time of the camera) and/or reducing the noise level depending on the array sensor technology. As an example, in the Fig. 3(a), we note that the luminescence at the very low pulse energy level of $0.4 \mathrm{~nJ}$ still leads to a well imaged microsplasma, provided that the exposure time and digital gain of the camera are increased in comparison to the higher energy

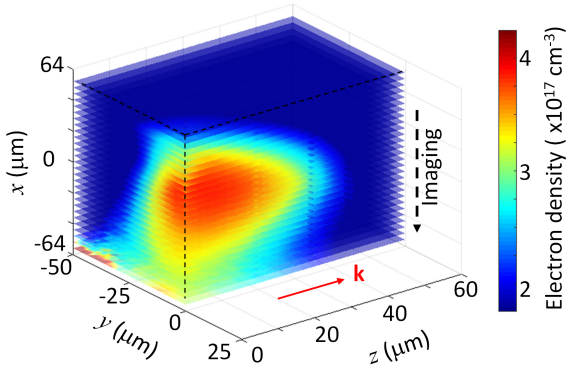

FIG. 4. 3D electrons density distribution of a prefocal plasma generated by femtosecond pulse focused at 0.3NA lens inside GaAs as retrieved by compensated luminescence images at different lateral depths. $Z=0$ represents the surface of the sample. The focal plane of the lens is at the depth of $300 \mu \mathrm{m}$ (z direction).

The laser propagation direction is marked by the vector $k$

cases. Using the same camera, it was impossible to detect any excitation at this energy level by shadowgraphy because the absorption at this excitation level is negligible. From this image, the minimum electron density that can be measured with these camera conditions is evaluated at $2 \times 10^{16} \mathrm{~cm}^{-3}$ (taking signal-to-noise ratio $>3$ as criterion). However, this limit is specific to the noise level of the standard InGaAs detector used for this measurement. This can be surely improved with superior performance imagers as for instance highly-cooled sensor arrays.

The results shown above demonstrate the possibility of using luminescence imaging to quantitatively analyze twodimensional electron density distributions, an aspect that was already exploited in semiconductor surface studies ${ }^{29}$, but applied here for cross-sectional analyses of laser interactions in the bulk. Interestingly, 3D reconstructions become also possible by acquiring images while scanning the region of interest along the $x$-axis (see Fig. 1). Obviously, the depth of field of the imaging system gives the resolution limit (longitudinal resolution) along the newly introduced dimension but this can be appropriately adjusted with the choice of the imaging microscope objective depending on the targeted observations. To explore this possibility, we concentrate on the $3 \mathrm{D}$ observation of the modest free-carrier density generated in the pre-focal region. This is widely reported by integrated or indirect measurements and simulation studies in laser writing configurations $\mathrm{s}^{1,8,9}$ but there was no direct observation to date. Targeting the observation of a relatively large plasma from the surface of GaAs, we use a lens of 0.3 NA (Olympus $10 \times$ ) for modest focusing conditions of the pump pulse. The weak focusing conditions does not allow to concentrate the pulse energy to the focal spot ${ }^{8,9}$. A schematic of the setup is shown in Fig. S3. The lateral imaging system is equipped with a lens of 0.65 NA (Olympus $50 \times$ ) for a good compromise between a large field of view and the depth of field $(\approx 18 \mu \mathrm{m}$ in this case) that defines the longitudinal resolution. It is also important to highlight that the collected signals from a large volume 
are affected by varying material absorption with the imaging depth. For a rigorous reconstruction, we have then compensated each image by dividing the signal by the absorption ratio described by the Beer Lambert law mentioned before. After this compensation, stacking the images leads to the 3D plasma density distribution shown in Fig. 4. Note that only half of the cylindrical-symmetry plasma is shown in the figure so that the displayed cross-section of the images showing the plasma core gives an image representation that should be similar to previous representations. However, we can note an asymmetry with an apparently larger plasma along the imaging axis. This is caused directly by the longitudinal resolution mentioned above that is comparable to the observed plasma dimensions $(<60 \mu \mathrm{m})$. Despite this artifact that is only depending on the chosen imaging conditions, the luminescence method performs well in displaying in 3D the free-carrier density distributions with a near-conical shape in the pre-focal region and which are not measurable in shadowgraphy studies ${ }^{9}$. Additionally, one can extrapolate that similar measurements made with a confocal microscopy arrangement would surely have improved the resolution for direct access to all the detailed spatial features of emission. However, 3D scanning procedures need longer times for image reconstruction.

For technological considerations, it is also important to highlight the simplicity of the luminescence method with no need of an additional synchronized beam as for pump-probe techniques. Therefore, it can offer a practical solution for rapid assessment of beam delivery and optimization of the applied conditions in laser writing configurations. For example, the focusing conditions in high-index semiconductors are strongly influenced by spherical aberrations that varies as a function of processing depth. The situation becomes even more complex when using intense beams that add nonlinear propagation distortions to the problem. By simply monitoring the peak luminescence, one can rapidly determine the optimum with the control parameters to achieve the strongest possible localization and excitation level.

An example of such an optimization procedure is shown in Fig. 5 where we show the dependence of distortions to the focusing depth. The correction collar is set for compensation at about 300-m depth and the lens is scanned to vary the processing depth. Alternatively, one could have set the depth and adjust gradually the correction collar for an analog change of the spherical aberration conditions. As shown in Fig. 5(a), as the focusing lens is shifted gradually from smaller to deeper depths (position 0 is defined arbitrarily), the luminescence gradually increases and then decreases. In the linear case, one predict a flip-flop of the asymmetry when crossing the optimum correction because such procedure would switch from over-compensation to under-compensation for spherical aberrations. However, we observe systematically carrot-like structures with the same orientation. This is due to the power of applied beam and shows clearly the dominance of plasma and nonlinear propagation effects to drive the focus features. In Fig. 5(b), the peak intensity from the collected luminescence images is plotted as a function of the focusing depth. Despite the complexity of the theoretical problem, it is interesting to see that a practical optimum position can be unambiguously

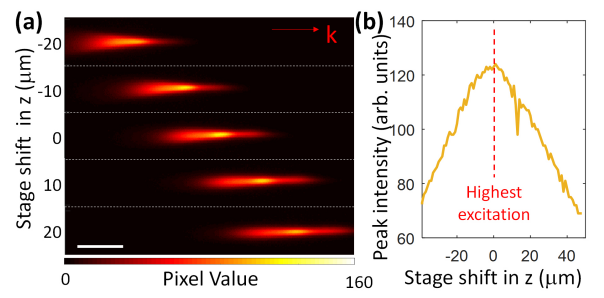

FIG. 5. Focusing depth dependence of the luminescence distributions generated with 190 -fs pulses focused with NA=0.85 in GaAs. (a) Luminescence images acquired for different stage shifts of the objective lens in $z$ direction shown in Fig. 1. The scale bar is $40 \mu \mathrm{m}$. (b) The peak value of the collected signals as a function of stage shifts.

found to achieve the maximum excitation level at the applied laser power level. While we have concentrated here on the optimization of the focusing depth for illustration purpose, the method can be generalized for optimization of other control parameters. This is of particular relevance for $3 \mathrm{D}$ writing in semiconductors with ultrashort pulses because of the severe requirements reported on the irradiation characteristics to exceed the threshold of modification. 1,30

In conclusion, we identified and measured band-to-band luminescence from microplasmas generated by ultrashort laser pulses inside GaAs and Si. This can offer a simple and robust method for quantitative evaluation of the excitation density inside the bulk semiconductor in experiments with tightly focused ultrashort pulses. Calibration can also be achieved via a reference measurement and does therefore not necessarily require the knowledge on the characteristics of the measuring system and the material properties. Compared to pump-probe techniques, the method avoids using an ultrafast pulse for illumination. The measurement is then not time-resolved, but the zero-background emission signal results in superior dynamic range and sensitivity, an aspect illustrated by the measurement of free-carrier densities as low as $2 \times 10^{16} \mathrm{~cm}^{-3}$ in GaAs. The methods can be easily implemented on a laser processing system allowing in situ optimization of the control parameters for the challenging problem of 3D laser writing inside semiconductors. Other potential applications of controlled microplasma generation are related to non-invasive diagnostics for defectivity and reliability analyses of microelectronics systems. Some advanced methods rely on the dynamical measurement of electrical signal responses influenced by laser stimulation ${ }^{31}$. Luminescence imaging is also exploited for all-optical analyses applicable to surfaces. ${ }^{29,32}$ The present study offers a unique possibility for space-resolved 3D diagnostics in this context.

\section{SUPPLEMENTARY MATERIALS}

See the supplementary materials for the following data: S1. Luminescence measurement in silicon. S2. The shadowgraphy images taken by z-scan of the imaging system. S3. The 
sketch of the 3D measurement process. S4. Calibration-free calculation of electron density through luminescence map. S5. Drude model to predict electrons density.

\section{ACKNOWLEDGMENTS}

This research has received funding from the European Research Council (ERC) under the European Union's Horizon 2020 research and innovation programme (grant agreement No 724480$)$.

\section{DATA AVAILABILITY}

The data that support the findings of this study are available from the corresponding author upon reasonable request.

${ }^{1}$ M. Chanal, V. Y. Fedorov, M. Chambonneau, R. Clady, S. Tzortzakis, an D. Grojo, "Crossing the threshold of ultrafast laser writing in bulk silicon," Nat. Commun. 8, 1-6 (2017).

${ }^{2}$ O. Tokel, A. Turnalı, G. Makey, P. Elahi, T. Çolakoğlu, E. Ergeçen, Ö. Yavuz, R. Hübner, M. Z. Borra, I. Pavlov, et al., "In-chip microstructures and photonic devices fabricated by nonlinear laser lithography deep inside silicon," Nature photonics 11, 639-645 (2017).

M. Chambonneau, Q. Li, M. Chanal, N. Sanner, and D. Grojo, "Writing waveguides inside monolithic crystalline silicon with nanosecond lase pulses," Opt. Lett. 41, 4875-4878 (2016)

${ }^{4}$ G. Matthäus, H. Kammer, K. A. Lammers, C. Vetter, W. Atanabe, S. N. Olte, G. Matthäus, H. Kämmer, K. A. Lammers, C. Vetter, W. Watanabe, and S. Nolte, "Inscription of silicon waveguides using picosecond pulses," Opt. Express 26, 24089-24097 (2018).

${ }^{5}$ I. Pavlov, O. Tokel, S. Pavlova, V. Kadan, G. Makey, A. Turnali, Ö. Yavuz, and F. Ö. Ilday, "Femtosecond laser written waveguides deep inside silicon," Opt. Lett. 42, 3028 (2017)

${ }^{6}$ A. Alberucci, N. Alasgarzade, M. Blothe, M. Chambonneau, H. Kämmer, G. Matthäus, C. P. Jisha, and S. Nolte, "In-depth optical characterization of femtosecond-written waveguides in silicon,” Phys. Rev. Appl. 14, 24078 (2020).

M. Chambonneau, D. Richter, S. Nolte, and D. Grojo, "Inscribing diffraction gratings in bulk silicon with nanosecond laser pulses," Opt. Lett. $\mathbf{4 3}$ 6069 (2018).

${ }^{8}$ V. V. Kononenko, V. V. Konov, and E. M. Dianov, "Delocalization of femtosecond radiation in silicon," Opt. Lett. 37, 3369 (2012).

${ }^{9}$ A. Mouskeftaras, A. V. Rode, R. Clady, M. Sentis, O. Utéza, and D. Grojo, "Self-limited underdense microplasmas in bulk silicon induced by ultrashort laser pulses," Appl. Phys. Lett. 105, 191103 (2014)

${ }^{10}$ M. Chambonneau, L. Lavoute, D. Gaponov, V. Y. Fedorov, A. Hideu S. Février, S. Tzortzakis, O. Uteza, D. Grojo, O. Utéza, and et al., "Competing nonlinear delocalization of light for laser inscription inside silicon with a 2-m picosecond laser," Phys. Rev. Appl. 12, 24009 (2019).

${ }^{11} \mathrm{~V}$. Kononenko, E. Zavedeev, and V. Gololobov, "The effect of light-induced plasma on propagation of intense fs laser radiation in c-si," Appl. Phys. A 122, 293 (2016).
${ }^{12}$ X. Yu, X. Wang, M. Chanal, C. A. Trallero-Herrero, D. Grojo, and S. Lei, "Internal modification of intrinsic and doped silicon using infrared nanosecond laser," Appl. Phys. A 122, 1-7 (2016).

${ }^{13}$ A. Wang, A. Das, and D. Grojo, "Ultrafast laser writing deep inside silicon with thz-repetition-rate trains of pulses," Research 2020 (2020).

${ }^{14} \mathrm{~V}$. Grivickas and J. Linnros, "Carrier lifetime: free carrier absorption, photoconductivity, and photoluminescence," Charac. Mater. , 1-35 (2002).

${ }^{15} \mathrm{~A}$. Gopal, S. Minardi, and M. Tatarakis, "Quantitative two-dimensional shadowgraphic set-up for high-sensitivity measurement of low-density laser-plasmas," Opt. Lett. 32, 1238-40 (2007).

${ }^{16}$ V. V. Kononenko, V. I. Konov, V. M. Gololobov, and E. V. Zavedeev, "Propagation and absorption of high-intensity femtosecond laser radiation in diamond," Quantum Electronics 44, 1099 (2014).

${ }^{17}$ A. Mouskeftaras, M. Chanal, M. Chambonneau, R. Clady, O. Utéza, and D. Grojo, "Direct measurement of ambipolar diffusion in bulk silicon by ultrafast infrared imaging of laser-induced microplasmas," Appl. Phys. Lett. 108, 041107 (2016)

${ }^{18}$ A. Das, A. Wang, O. Uteza, and D. Grojo, "Pulse-duration dependence of laser-induced modifications inside silicon," Opt. Express 28, 26623-26635 (2020)

${ }^{19}$ V. Y. Fedorov, M. Chanal, D. Grojo, and S. Tzortzakis, "Accessing extreme spatiotemporal localization of high-power laser radiation through transformation optics and scalar wave equations," Phys. Rev. Lett. 117, 43902 (2016)

${ }^{20}$ S. Kudryashov, N. Stsepuro, P. Danilov, N. Smirnov, A. Levchenko, and M. Kovalev, "Cumulative defocusing of sub-mhz-rate femtosecond-laser pulses in bulk diamond envisioned by transient a-band photoluminescence," Optical Materials Express 11, 2234-2241 (2021).

${ }^{21}$ F. Dreisow, M. Heinrich, A. Szameit, S. Döring, S. Nolte, A. Tünnermann, S. Fahr, and F. Lederer, "Spectral resolved dynamic localization in curved fs laser written waveguide arrays," Optics express 16, 3474-3483 (2008).

${ }^{22}$ A. Mermillod-Blondin, C. Mauclair, A. Rosenfeld, J. Bonse, I. V. Hertel, E. Audouard, and R. Stoian, "Size correction in ultrafast laser processing of fused silica by temporal pulse shaping," Applied Physics Letters 93, 021921 (2008)

${ }^{23}$ C. Carr, H. Radousky, A. Rubenchik, M. Feit, and S. Demos, "Localized dynamics during laser-induced damage in optical materials," Physical review letters 92, 087401 (2004).

${ }^{24}$ J. Lohani, S. Yadav, R. Tyagi, and S. Sapra, "Efficient fluorescence quenching of cdse quantum dots on epitaxial gaas nanostructures," J. of Nanoparticle Research 21, 1-12 (2019).

${ }^{25} \mathrm{M}$. D. Sturge, "Optical absorption of gallium arsenide between 0.6 and 2.75 ev," Phys. Rev. 127, 768-773 (1962)

${ }^{26}$ W. Van Roosbroeck and W. Shockley, "Photon-radiative recombination of electrons and holes in germanium," Phys. Rev. 94, 1558 (1954).

${ }^{27}$ U. Strauss, W. Rühle, and K. Köhler, "Auger recombination in intrinsic gaas," Appl. Phys. Lett. 62, 55-57 (1993).

${ }^{28} \mathrm{D}$ Grojo, S. Leyder, P. Delaporte, W. Marine, M. Sentis, and O. Utéza, "Long-wavelength multiphoton ionization inside band-gap solids," Physical Review B 88, 195135 (2013).

${ }^{29}$ T. Trupke, R. Bardos, M. Schubert, and W. Warta, "Photoluminescence imaging of silicon wafers," Appl. Phys. Lett. 89, 044107 (2006)

${ }^{30}$ A. Wang, A. Das, and D. Grojo, "Temporal-contrast imperfections as Arivers for ultrafast laser modifications in bulk silicon," Phys. Rev. Res. 2, $033023(2020)$

${ }^{31}$ M. Chambonneau, S. Souiki-Figuigui, P. Chiquet, V. Della Marca, J. PostelPellerin, P. Canet, J.-M. Portal, and D. Grojo, "Suppressing the memory state of floating gate transistors with repeated femtosecond laser backside irradiations," Appl. Phys. Lett. 110, 161112 (2017).

${ }^{32} \mathrm{~J}$. Giesecke, M. Kasemann, and W. Warta, "Determination of local minority carrier diffusion lengths in crystalline silicon from luminescence images," J. Appl. Phys. 106, 014907 (2009). 


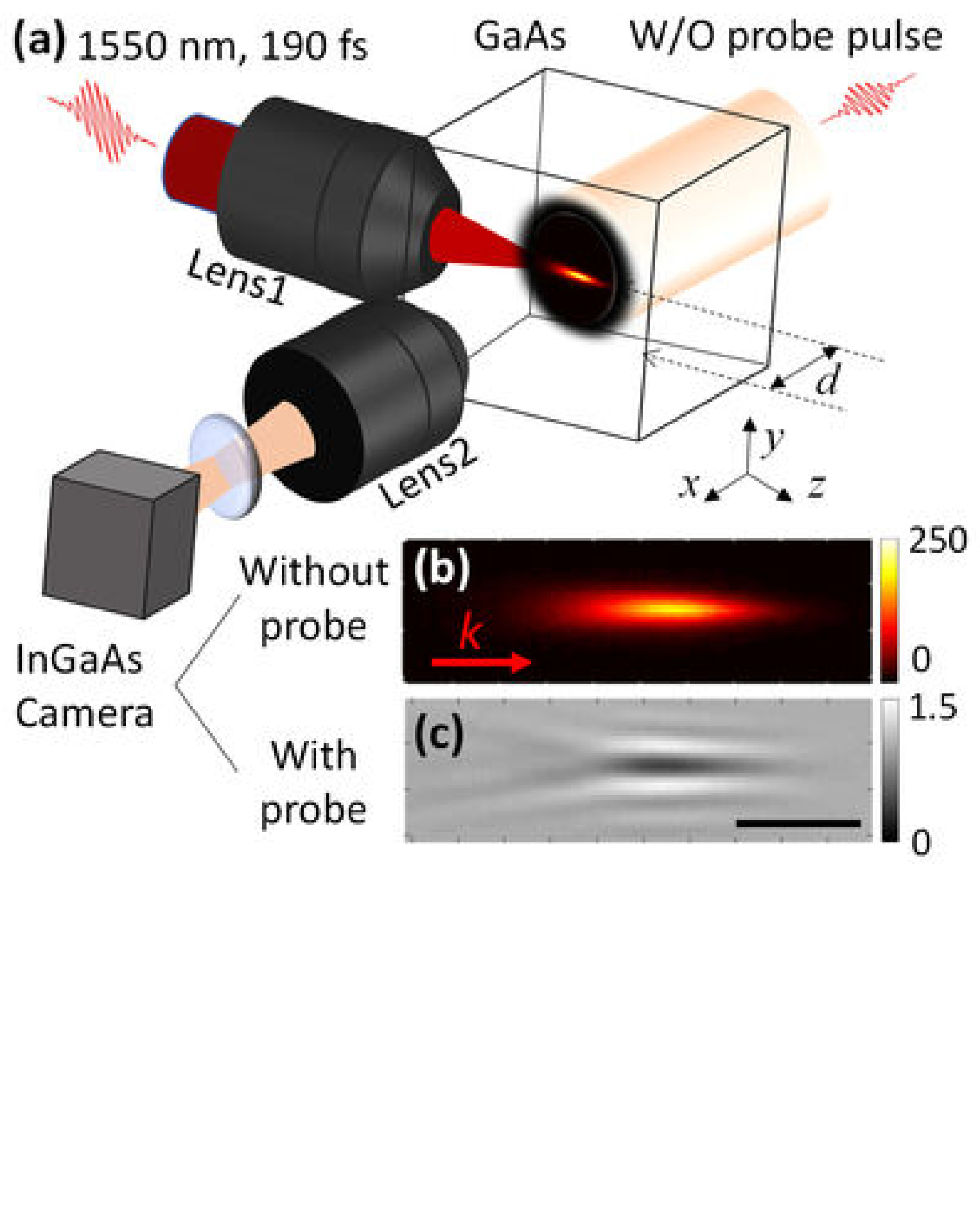



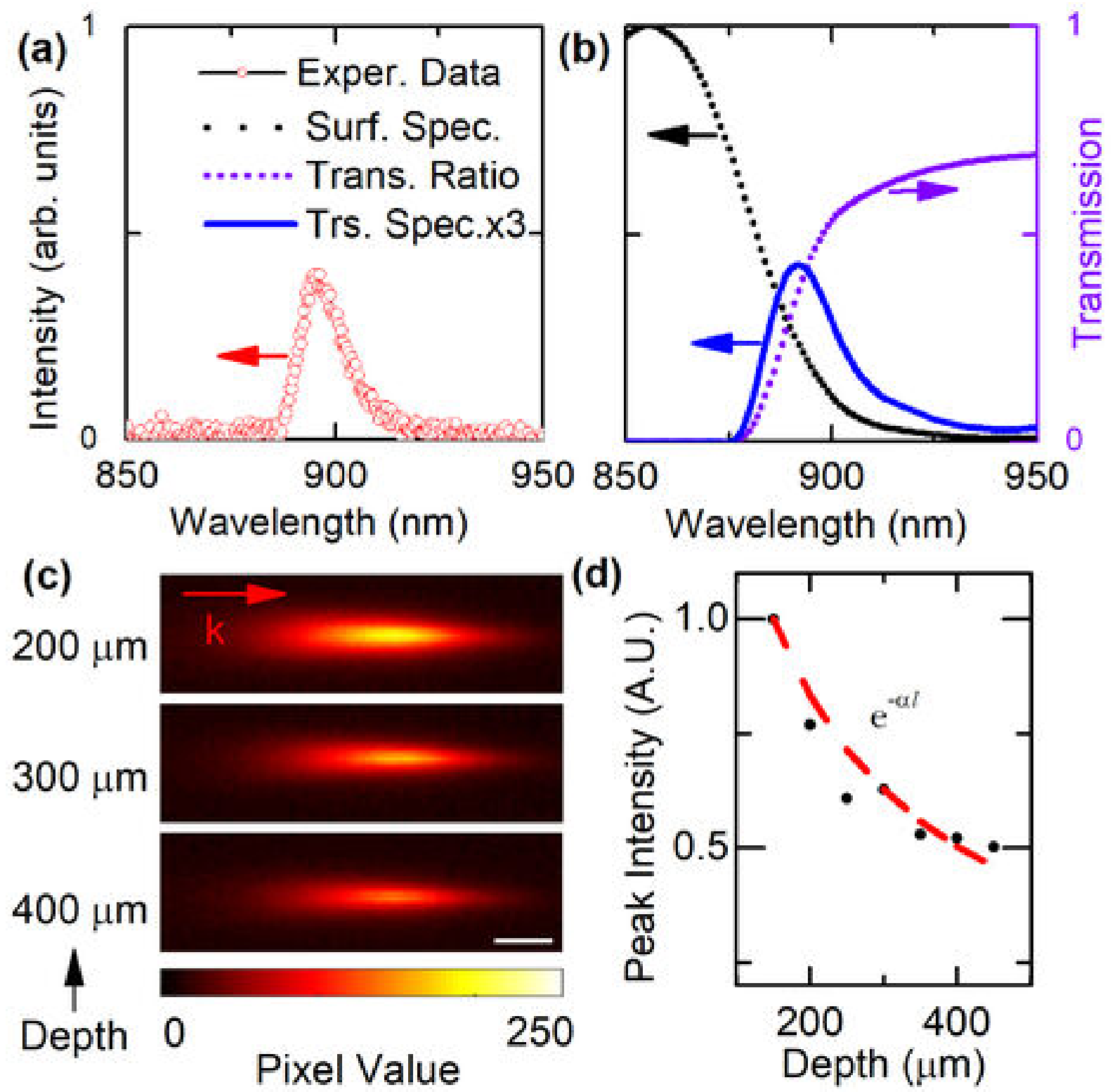

(d)

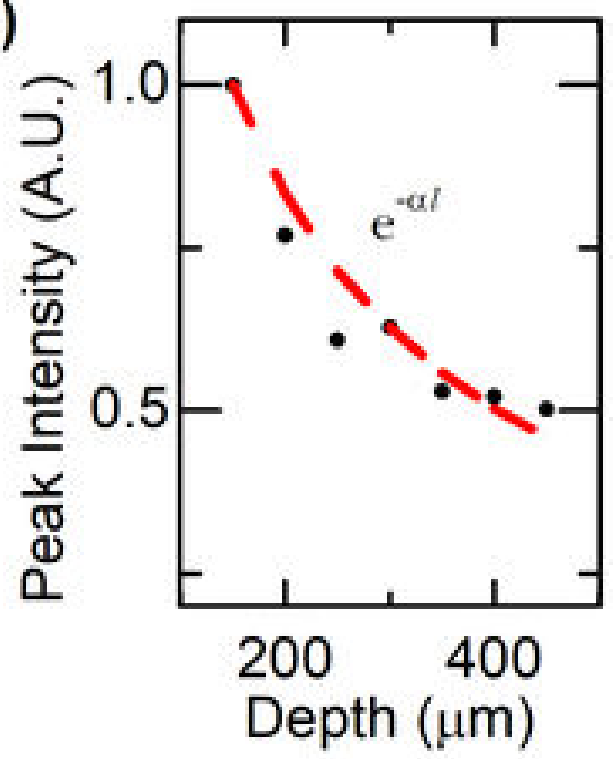



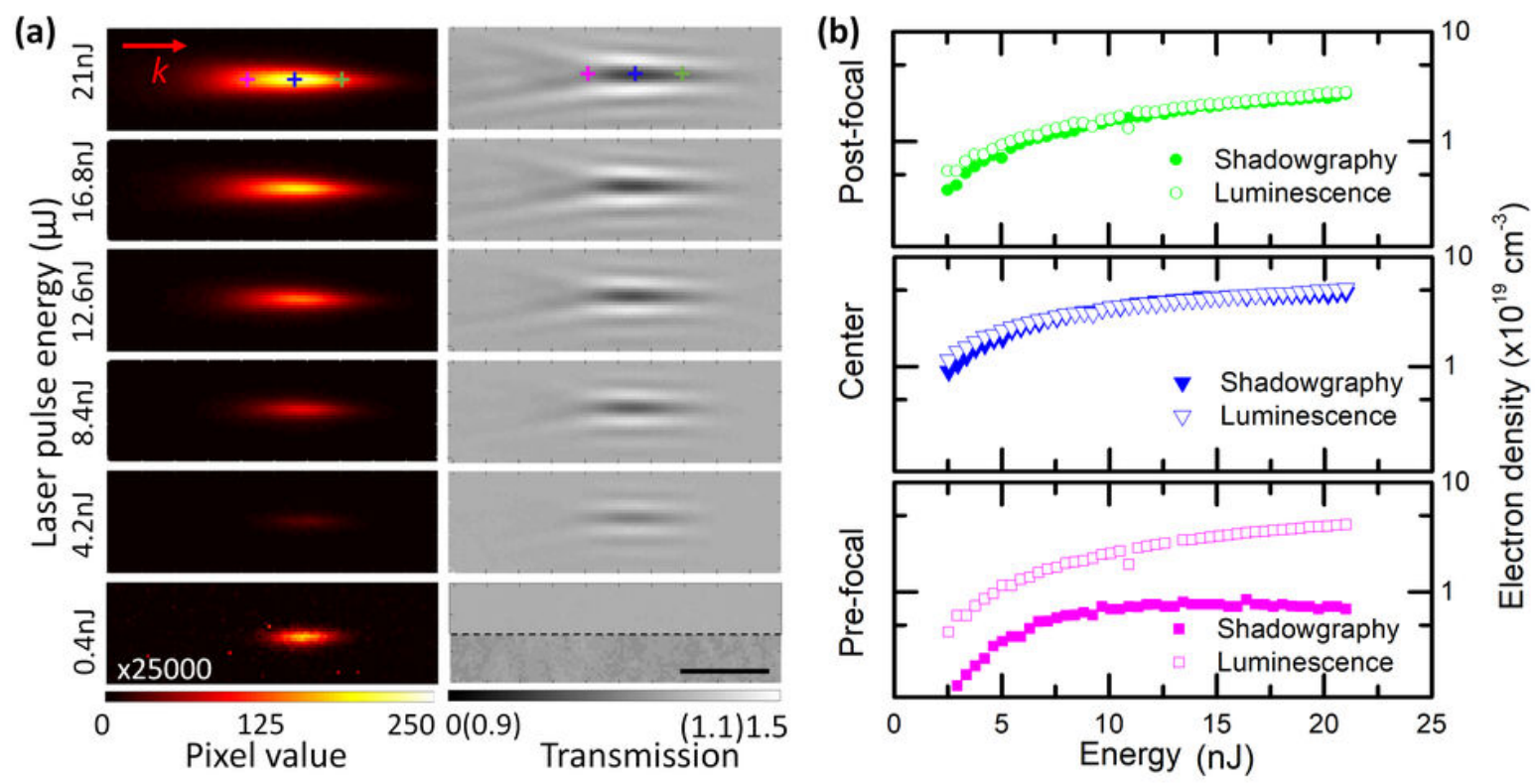


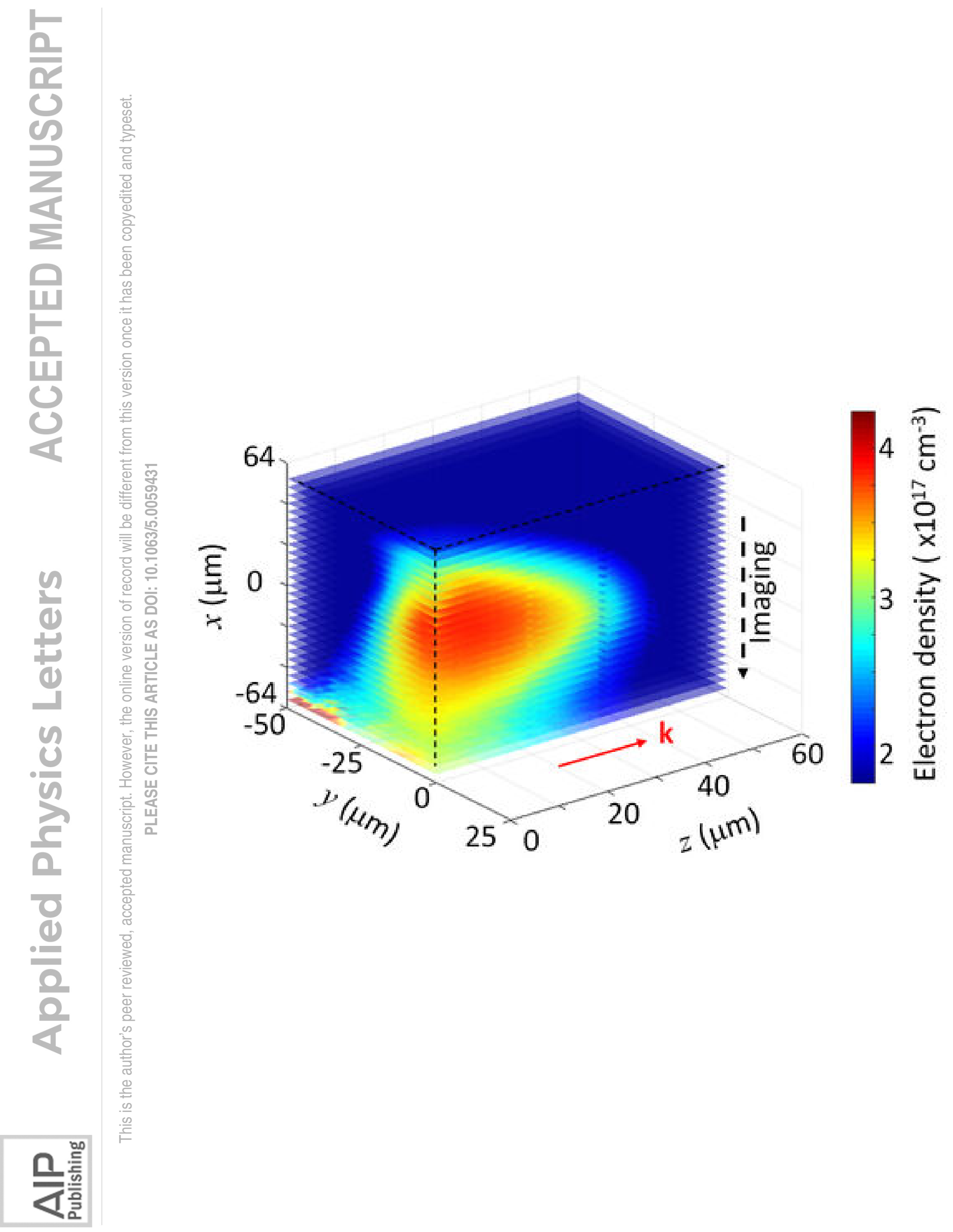




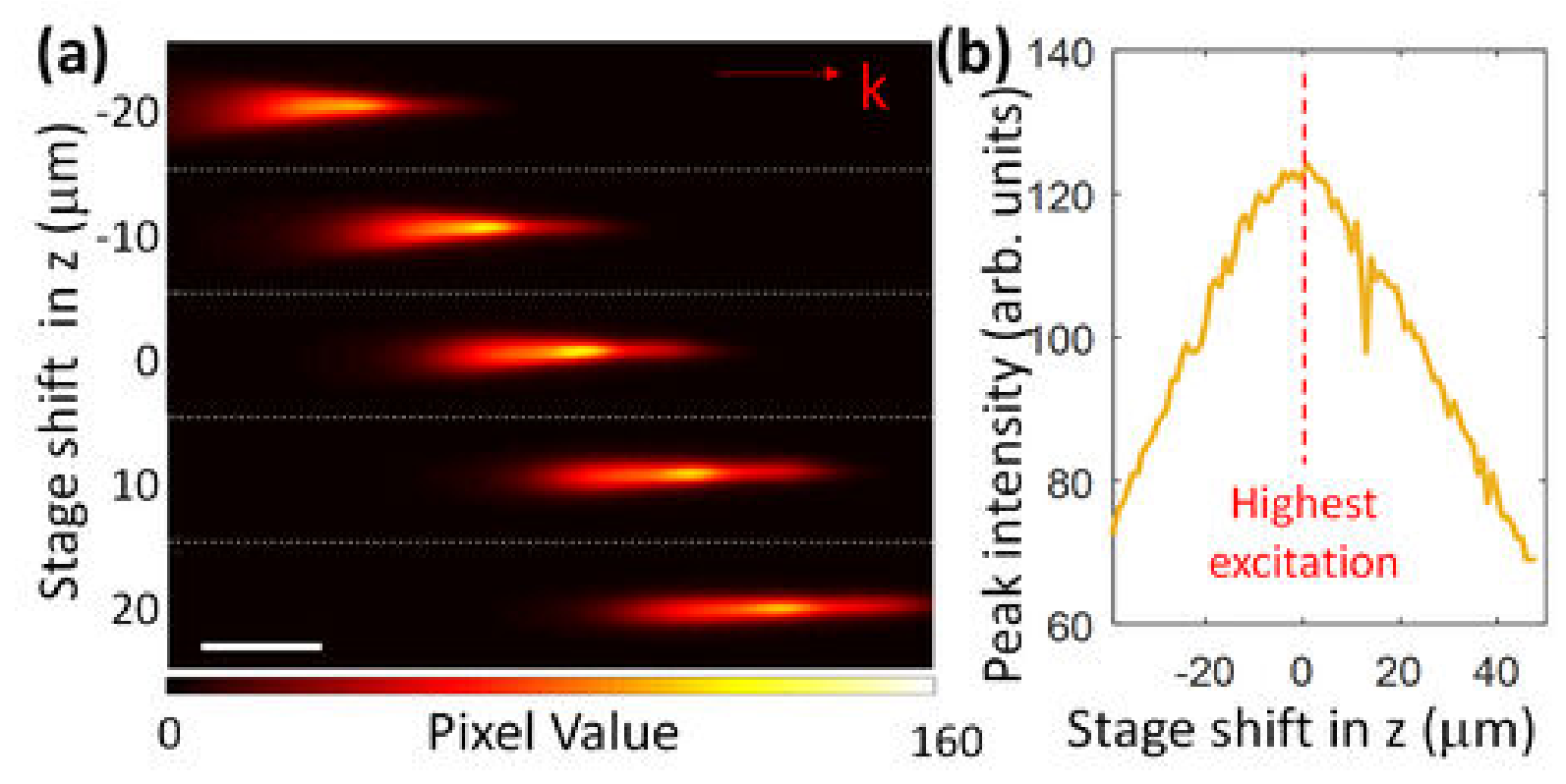

